\title{
AN ANALYSIS OF THE GENERALIZED DOUBLE-SLIDING MODELS FOR COHESIONLESS GRANULAR MATERIALS
}

\author{
N. P. KRUYT \\ Department of Mechanical Engineering. University of Twente. Enschede, The Netherlands
}

(Received 24 Notenter $1988:$ in revised form 3 March 1989)

\begin{abstract}
A WELL-KNOWN class of constitutive relations for cohesionless granular materials are the double-sliding models. The double-sliding model proposed by Spencer $(J$. Mech. Phys. Soldds, 12, 337, 1964) for incompressible materials as well as those of SPENCER and KINGSTON (Rheol. Acta, 12, 194, 1974) and MeHrabad and Cown (J. Mech. Phys. Solids. 26, 269. 1978) for compressible materials are generafized to describe the unsteady fow of cohesionless granular materials. These models anc analysed and it is shown that they lead to equations that are not well-posed. Implications of this finding are discussed,
\end{abstract}

\section{INTRODUCTION}

THE STRESSES in cohesionless granular materials are reasonably well deseribed by the Coulomb yield criterion. Various theories have been proposed to describe the kinematic behaviour of granular materials. A well-known model for large, continued deformations in two dimensions was developed by SPENCER (1964).

It postulates that the material is isotropic and incompressible and satisfies the Coulomb yield criterion. The equations that govern the kinematic behaviour are derived from physically plausible assumptions. The deformation is assumed to occur by a superposition of two simple shearing motions along the sliplines (where the critical ratio of shear to normal stress is attained) and a rotation of the slipline field. It was from these principles that SPENCER (1964) derived his so-ealled velocity equation.

In Spencer's original model it is assumed that the material is incompressible. Various generalizations have been proposed to include the effect of the compressibility of the material: SPENCER and KingsTon (1974) and MeHRABADI and CowIN (1978). In its simplest form the proposal of SPENCER and KINGSTON (1974) states that the density is determined by the mean pressure, whereas MFHRABADI and CowIN (1978) included the effect of the dilatancy, i.e. the volume change induced by shear deformation, into the double-sliding model.

In contrast to plasticity relations based on a smooth flow potential the doublesliding models predict that the stress and deformation-rate tensors are non-coaxial. Experimental evidence (Drescher and DE Josselin De JoNG, 1972; OdA and KoNISHI, 
1974 ; DresCher, 1976 ; Allersma, 1985) confirms the existence of this non-coaxiality (at least under certain conditions).

Spencer's incompressible model has been generalized by MORRISON and RICHMOND (1976) to describe the unsteady flow of granular materials. The same will be done here for both models mentioned that include the compressibility of the material.

The complete system of equations that will be studied here consists of the momentum equations. the continuity equation, the Coulomb yield criterion, the velocity equation and a density equation.

It will be shown that these equations do not satisfy the Hadamard stability criterion (JOSEPH et al., 1985), which requires that plane-wave solutions remain bounded as $t \rightarrow \infty$. This means that the double-sliding models are not well-posed. In the case of Spencer's original incompressible model this was previously stated by SCHAFFFFR (1987).

Tensile stresses will be reckoned as positive, this being the usual convention in continuum mechanics. The stress tensor is denoted by $\mathbf{T}$ and the velocity vector by $\mathbf{u}=(u, v)^{T}$. The rate of deformation tensor $\mathbf{D}$ and the vorticity tensor $\mathbf{W}$ are defined by

$$
\begin{aligned}
\mathbf{D} & =\frac{1}{2}\left[(\nabla \mathbf{u})+(\nabla \mathbf{v})^{T}\right], \\
\mathbf{W} & =\frac{1}{2}\left[(\nabla \mathbf{u})-(\nabla \mathbf{u})^{T}\right] .
\end{aligned}
$$

In two dimensions the deviator $\mathbf{D}^{\prime}$ of $\mathbf{D}$ is given by

$$
\mathbf{D}^{\prime}=\mathbf{D}-\frac{1}{2}(\operatorname{tr} \mathbf{D}) \mathbf{1} \text {. }
$$

The deviator $\mathbf{T}^{\prime}$ of the stress tensor is similarly defined. The mean pressure $p$ is given by

$$
p=-\frac{1}{2} \operatorname{tr} \mathbf{T}
$$

and the invariant $\tau$ of the stress deviator by

$$
\tau^{2}=\frac{1}{2} \operatorname{tr}\left(\mathbf{T}^{\prime} \cdot \mathbf{T}^{\prime}\right)
$$

\section{The Basic Equations and the Coulomb Yield Criterion}

The momentum equations in the vertical and horizontal direction are respectively

$$
\rho\left[\frac{\partial u}{\partial t}+u \frac{\partial u}{\partial x}+v \frac{\partial u}{\partial y}\right]=\frac{\partial T_{x x}}{\partial x}+\frac{\partial T_{x y}}{\partial y}+\rho g
$$

and

$$
\rho\left[\frac{\partial v}{\partial t}+u \frac{\partial v}{\partial x}+v \frac{\partial v}{\partial y}\right]=\frac{\partial T_{y y}}{\partial x}+\frac{\partial T_{y y}}{\partial y} .
$$

The continuity equation reads 


$$
\frac{\partial \rho}{\partial t}+u \frac{\partial \rho}{\partial x}+r \frac{\partial \rho}{\partial y}+\rho\left[\frac{\partial u}{\partial x}+\frac{\partial v}{\partial y}\right]=0 .
$$

It is assumed that the material is cohesionless and satisfies the Coulomb yield criterion, which can be expressed in the Sokolovski variables $\psi$ and $p$ as

$$
\begin{aligned}
& T_{x x}=-p[1-\sin (\phi) \cos (2 \psi)] . \\
& T_{y y}=-p[1+\sin (\phi) \cos (2 \psi)], \\
& T_{x y}=p \sin (\phi) \sin (2 \psi) .
\end{aligned}
$$

Here $\psi$ is the angle of the direction of the algebraically greater principal stress with the (vertical) $x$-axis and $\phi$ is the internal friction angle.

\section{The Kinematic BehaViour}

The generalized double-sliding models consist of two additional equations that describe the kinematic behaviour: a velocity equation and a density equation.

The generalized velocity equation as proposed by MeHRABaDI and CowIN (1980) is

$$
\mathbf{T} \cdot \stackrel{\mathbf{T}}{-} \mathbf{T} \cdot \mathbf{T}=2 h_{1}[\mathbf{T} \cdot \mathbf{D}-\mathbf{D} \cdot \mathbf{T}] .
$$

In (3.1) $\uparrow$ denotes the objective Jaumann derivative

$$
\mathbf{T}=\mathbf{T}-\mathbf{W} \cdot \mathbf{T}+\mathbf{T} \cdot \mathbf{W} .
$$

In (3.2) the superimposed dot denotes the material time derivative. The parameter $h_{1}$ in (3.1) is the so-called non-coaxiality parameter. It is a measure of the non-coaxiality between the stress and deformation-rate tensors. In scalar form (3.1) reads

$$
\dot{\psi}+W_{x y}=\frac{h_{1}}{2 \tau}\left[2 \cos (2 \psi) D_{x y}-\sin (2 \psi)\left(D_{x x}-D_{y y}\right)\right] .
$$

Two density equations have been proposed : a simple relation between density and pressure was given by SPENCER and KINGSTON (1974) and a more sophisticated dilatancy relation by MEHRABADI and CowIN (1978).

SPENCER and KINGSTON (1974) proposed the following relation:

$$
\frac{\mathrm{d} \rho}{\mathrm{d} p}=F\left(\rho, p, k_{H}\right) .
$$

Here $k_{H}$ denotes a hardening parameter that describes the stress and deformation history. In its simplest form, if the effect of the stress and deformation history is neglected, (3.4) reads

$$
\rho=f(p) .
$$

It will be assumed here that the density is a strictly increasing function of the mean pressure $p$. 
The Mehrabadi-Cowin dilatancy relation was derived by MrHRABaDi and CowTN (1978) and HARRIS (1985). It gives a relation between the volume increase induced by shear deformation and the distortional work. This dilatancy equation is

$$
\tau \operatorname{tr}(\mathbf{D})=\beta \operatorname{tr}\left(\mathbf{T}^{\prime} \cdot \mathbf{D}^{\prime}\right) .
$$

The dilatancy parameter $\beta$ is defined by $\beta=\sin (v) / \cos (\phi-v)$. where $v(v \leqslant \phi)$ is the dilatancy angle. The original dilatant double-sliding model of MEHRABADI and COWIN (1978) is retrieved if $h_{1}$ in (3.1) is given by

$$
\frac{h_{1}}{\tau}=\frac{1-\beta \sin (\phi)}{\beta-\sin (\phi)} .
$$

The incompressible model of SPENCER (1964) is a special case of the MehrabadiCowin model : it is obtained if $\mathrm{v}=0$ and hence $\beta=0$.

\section{Analysis of the Generalized Spencer-Kingston Modfi.}

The generalization of the Spencer-Kingston double-sliding model to the unsteady flow of cohesionless granular materials consists of (2.1)-(2.6), (3.3) and (3.5). Symbolically these equations can be written, after the substitution of (3.5) into (2.3), as

$$
\frac{\partial}{\partial t}\left[\begin{array}{c}
u \\
v \\
\psi \\
p
\end{array}\right]=\mathbf{A}(\mathbf{w}) \frac{\partial \mathbf{w}}{\partial \mathbf{x}}+\mathbf{B}(\mathbf{w}) \frac{\partial \mathbf{w}}{\partial \mathbf{y}}+\left[\begin{array}{l}
g \\
0 \\
0 \\
0
\end{array}\right] .
$$

where $\mathbf{w}=(u, v, \psi, p)$. The matrices $\mathbf{A}$ and $\mathbf{B}$ are given in Appendix $\mathbf{A}$.

A plane-wave solution will be substituted into the system of equations (4.1). This plane-wave solution has the form

$$
\mathbf{w}=\mathbf{w}_{0} \exp [\mathrm{i}(\omega t+\mathbf{k} \cdot \mathbf{x})]
$$

where $\mathbf{k}=(k \cos (\vartheta), k \sin (\vartheta))^{T}$ and $\mathbf{x}=(x, y)^{T}$.

Disregarding the source term it follows from (4.1) that non-trivial plane-wave solutions exist if and only if

$$
\operatorname{det}\left[\cos (\vartheta) \mathbf{A}+\sin \left(\dot{(j)} \mathbf{B}-{ }_{k}^{(j)} \mathbf{I}\right]=0,\right.
$$

where $I$ is the unit matrix.

Equation (4.3) can be solved for w. If all solutions are real (for all 9) then the system of equations is hyperbolic. However, if for some it, one of the solutions has a negative imaginary part, then the problem is not well-posed. In this case the solution can grow exponentially with time, as can be seen from (4.2). This means that the equations do not satisfy the Hadamard stability criterion. 
The solutions for $\omega$ are given in Appendix B, where it is also shown that no value of $h_{1}$ exists for which the Hadamard stability condition is satisfied. This means that the Spencer-Kingston double-sliding models are not well-posed.

\section{Analysis of the Generalized Mehrabadi-Cowin Model}

The generalization of the Mehrabadi-Cowin double-sliding model to the unsteady flow of cohesionless granular materials consists of (2.1)-(2.6), (3.3) and (3.6). Symbolically these equations can be written as

$$
\frac{\partial}{\partial t}\left[\begin{array}{c}
u \\
v \\
\psi \\
\rho \\
0
\end{array}\right]=\mathbf{C}(\mathbf{w}) \frac{\partial \mathbf{w}}{\partial \mathbf{x}}+\mathbf{D}(\mathbf{w}) \frac{\partial \mathbf{w}}{\partial \mathbf{y}}+\left[\begin{array}{l}
g \\
0 \\
0 \\
0 \\
0
\end{array}\right],
$$

where $\mathbf{w}=(u, v, \psi, \rho, p)$. The matrices $\mathbf{C}$ and $\mathbf{D}$ are given in Appendix $\mathbf{C}$.

Again a plane-wave solution will be substituted into the system of equations. This plane-wave solution has the form

$$
\mathbf{w}=\mathbf{w}_{0} \exp [\mathbf{i}(\omega t+\mathbf{k} \cdot \mathbf{x})]
$$

where $\mathbf{k}=(k \cos (\vartheta), k \sin (\vartheta))^{T}$ and $\mathbf{x}=(x, y)^{T}$.

Disregarding the source term it follows that non-trivial plane-wave solutions exist if and only if

$$
\operatorname{det}\left[\cos (\vartheta) \mathbf{C}+\sin (\vartheta) \mathbf{D}-\frac{\omega}{k} \tilde{\mathbf{I}}\right]=0
$$

where the modified unit matrix $\tilde{I}$ is defined by

$$
\tilde{I}_{i j}=\left[\begin{array}{ll}
1 & \text { if } i=j \text { and } i<5 \\
0 & \text { otherwise. }
\end{array}\right.
$$

The Hadamard stability condition again requires that the imaginary part of the solutions for $\omega$ is never negative.

The solutions of (5.3) for $(1)$ are given in Appendix D, where it is also shown that no value of $h_{1}$ exists for which the Hadamard stability condition is satisfied. This means that the dilatant double-sliding models are not well-posed. Therefore the statement of MORRISON and RICHMOND (1976), that the generalization of Spencer's original model to the unsteady flow of granular materials leads to a hyperbolic system of equations, is incorrect. This was also remarked by SCHAEFFER (1987). 


\section{Discussion}

It has been shown that the incompressible and dilatant double-sliding models do not satisfy the Hadamard stability condition. This means that, according to these models, the material always shows unstable behaviour. For a simple shearing flow this was also shown by SPENCER (1986).

Stable flows of granular materials are physically possible, although they may be difficult to produce experimentally as there is a tendency for the formation of shear bands. Hence it is concluded that the deformation behaviour of granular materials is not described satisfactorily by the double-sliding models.

Apparently the double-sliding models are too restrictive. Possibly a better description of the material behaviour is given by the double-sliding free-rotating models of DE Josselin De Jong $(1971,1977)$. In these models (3.3) is replaced by

$$
\Omega+W_{x y}=\frac{h_{1}}{2 \tau}\left[2 \cos (2 \psi) D_{x y}-\sin (2 \psi)\left(D_{x x}-D_{y y}\right)\right] .
$$

where $h_{1}$ is given by (3.7) and $\Omega$ is an undetermined rotation rate.

Some experimental support for these models is given by DrEsCHER (1976). A disadvantage of the free-rotating double-sliding models is the indeterminacy of the rotation rate $\Omega$, which makes it impossible to perform definite calculations.

Micro-mechanical modelling (CHRISTOFFERSEN et al., 1981) could lead to a relation between $\Omega$ and other quantities describing the stresses and deformation of the granular material. An alternative to this approach is the numerical modelling of assemblies of particles (Cundall and SrRack, 1979). In this way it may be possible to obtain a model satisfying the Hadamard stability condition, but lacking the indeterminacy of the free-rotating double-sliding model.

\section{ACKNOWLEDGEMENT}

The author would like to thank a referee for drawing his attention to the 1986 article by Spencer.

\section{REFERENCES}

Allersma, H. G. B.

ChristofFERSEN, J., Mchrabad, M. M. and NEMAT-NASSER, $S$.

CUNDALL, P. A. and

STRACX. O. D. L.

DE JOSSELIN DE JONG ${ }_{4} G$.

DE JosSELIN DE JoNG, G.

Drescher, A.

DRESCHER, A. and

De Josselin De JoNG, G.
1985 J. Powder Bulk Solids Tech. 9, 7.

1981 J. Appl. Mech. 48, 339.

1979 Geotechique 29, 47.

1971 Geotechnique 21, 155.

1977 Arch. Mech. 29, 561.

1976 Geotechnique 26, 591 .

1972 J. Mech. Phis. Solids 20,337. 
HARRIS, D.

Joseph, D. D, Renardy, M. and SAUT, J. C.

Mehrabadi, M. M. and Cowin, S. C.

MehrabaDi, M. M. and CowIN, S. C.

MORRISON, H. L. and RICHMOND, $\mathrm{O}$.

ODA, M. and Konishi, J.

SCHAEFFER, D. G.

SPENCER, A. J. M.

SPENCER, A. J. M.

SPENCER, A. J. M. and KINGSTON, M. R.
1985 J. Mech. Phys. Solids 33, 51.

1985 Archs ration. Mech. Analysis

1978 J. Mech. Phys. Solids 26, 269.

1980 J. Engng Mech. Dit. Am. Soc. Cir. Engrs 106, 991.

1976 J.appl. Mech. 43, 49.

1974 Soils Foundations 14, 25.

1987 J. Diff. Eqns 66, 19.

1964 J. Mech. Phy's. Solids 12, 337.

1986 Acta Mechanica 64, 77.

1974 Rheol. Acta 12, 194.

\section{APPENDIX A}

The matrices $\mathbf{A}$ and $\mathbf{B}$ from the system of equations (4.1) are given by

$$
\mathbf{A}=\left[\begin{array}{cccc}
-u & 0 & -2 \frac{p}{\rho} \sin (\phi) \sin (2 \psi) & \frac{-1+\sin (\phi) \cos (2 \psi)}{\rho} \\
0 & -u & 2 \frac{p}{\rho} \sin (\phi) \cos (2 \psi) & \frac{1}{\rho} \sin (\phi) \sin (2 \psi) \\
\frac{-h_{1}}{2 \tau} \sin (2 \psi) & \frac{1}{2}+\frac{h_{1}}{2 \tau} \cos (2 \psi) & -u & 0 \\
\frac{-\rho}{\rho} & 0 & 0 & -u
\end{array}\right]
$$

and

$$
\mathbf{B}=\left[\begin{array}{cccc}
-v & 0 & 2 \frac{p}{\rho} \sin (\phi) \cos (2 \psi) & \frac{1}{\rho} \sin (\phi) \sin (2 \psi) \\
0 & -v & 2 \frac{p}{\rho} \sin (\phi) \sin (2 \psi) & \frac{-1-\sin (\phi) \cos (2 \psi)}{\rho} \\
-\frac{1}{2}+\frac{h_{1}}{2 \tau} \cos (2 \psi) & \frac{h_{1}}{2 \tau} \sin (2 \psi) & -v & 0 \\
0 & \frac{-\rho}{\rho} & 0 & -v
\end{array}\right],
$$

where $\rho^{\prime}$ is $\mathrm{d} \rho / \mathrm{d} \rho$.

\section{APPENDIX B}

Define $z=-(\omega / k)-\cos (\vartheta) u-\sin (\vartheta) v$.

It follows after some lengthy algebra that the solutions of (4.3) are determined from

$$
z^{4}-A z^{2}+B=0
$$

where 


$$
A=\frac{p}{\rho} \sin (\phi)\left[\frac{h_{1}}{\tau}+\cos (2 \psi-2 \theta)\right]+\frac{1}{\rho} \cdot[1-\sin (\phi) \cos (2 \psi-2 \gamma)]
$$

and

$$
B=\frac{p}{\rho \rho^{\prime}} \sin (\phi)\left[1+\frac{h_{1}}{\tau} \cos (2 \psi-29)\right][\cos (2 \psi-29)-\sin (\phi)] .
$$

So $z$ is determined from

$$
z^{2}={ }_{2}^{1}\left[A \pm\left(A^{2}-4 B\right)^{12}\right] \text {. }
$$

The solutions for $z$ have a non-negative imaginary part if and only if: $A \geqslant 0, B \geqslant 0$ and $A^{2}-4 B \geqslant 0$. It is impossible to find $h_{1}$ such that all solutions for $z$ are always real or have a positive imaginary part. This means that the generalized Spencer-Kingston double-sliding model always exhibits Hadamard instabilities.

\section{APPENDIX $\mathrm{C}$}

The matrices $\mathbf{C}$ and $\mathbf{D}$ from the system of equations (5.1) are given by

$$
\mathbf{C}=\left[\begin{array}{ccccc}
-u & 0 & -2 \frac{p}{\rho} \sin (\phi) \sin (2 \psi) & 0 & -1+\sin (\phi) \cos (2 \psi) \\
0 & -u & 2 \frac{p}{\rho} \sin (\phi) \cos (2 \psi) & 0 & \frac{1}{\rho} \sin (\phi) \sin (2 \psi) \\
\frac{-h_{1}}{2 \tau} \sin (2 \psi) & \frac{1}{2}+\frac{h_{1}}{2 \tau} \cos (2 \psi) & -u & 0 & 0 \\
-\rho & 0 & 0 & -u & 0 \\
1-\beta \cos (2 \psi) & -\beta \sin (2 \psi) & 0 & 0 & 0
\end{array}\right]
$$

and

$$
\mathbf{D}=\left[\begin{array}{ccccc}
-v & 0 & 2^{p} \sin (\phi) \cos (2 \psi) & 0 & \begin{array}{c}
1 \\
\rho
\end{array} \sin (\phi) \sin (2 \psi) \\
0 & -v & 2 \frac{p}{\rho} \sin (\phi) \sin (2 \psi) & 0 & -1-\sin (\phi) \cos (2 \psi) \\
-\frac{1}{2}+\frac{h_{1}}{2 \tau} \cos (2 \psi) & \frac{h_{1}}{2 \tau} \sin (2 \psi) & -v & 0 & \rho \\
0 & -\rho & 0 & -v & 0 \\
-\beta \sin (2 \psi) & 1+\beta \cos (2 \psi) & 0 & 0 & 0
\end{array}\right] .
$$

\section{APPENDIX D}

Define $z=-(\omega / k)-\cos (9) u-\sin (9) u$.

It follows after some lengthy algebra that the solutions of (5.3) are determined from

$$
C z^{3}=D z \text {, }
$$

where

$$
C=[(1+\beta \sin (\phi))-(\sin (\phi)+\beta) \cos (2 \psi-29)]
$$

and 


$$
D=2 \frac{p}{\rho} \sin (\phi)[\sin (\phi)-\cos (2 \psi-2 \theta)]\left[-\frac{1}{2}+\frac{h_{1}}{2 \tau} \beta+\left(\frac{1}{2} \beta-\frac{h_{1}}{2 \tau}\right) \cos (2 \psi-2 \vartheta)\right] .
$$

Since $v \leqslant \phi$ it follows that $C \geqslant 0$. The solutions for $z$ have a non-negative imaginary part if and only if $D \geqslant 0$, which is only possible if

$$
\left[-\frac{1}{2}+\frac{h_{1}}{2 \tau} \beta+\left(\frac{1}{2} \beta-\frac{h_{1}}{2 \tau}\right) \cos (2 \psi-2 \vartheta)\right]=K[\sin (\phi)-\cos (2 \psi-2 \vartheta)],
$$

where $K$ should be positive. It follows from (D4) that

$$
\frac{h_{1}}{\tau}=\frac{1-\beta \sin (\phi)}{\beta-\sin (\phi)} \text { and } \frac{h_{1}}{\tau}-\beta>0 \text {. }
$$

Hence it is impossible to find $h_{1}$ such that all solutions $z$ are always real or have a positive imaginary part. This means that the generalized dilatant double-sliding model always shows Hadamard instabilities. 